\title{
Desenvolvimento de um software educacional baseado na internet para estudos de casos clínicos.
}

\author{
Heloísa Melo Hertzog* \\ Lourenço de Oliveira Basso** \\ Dinarte Alexandre Prietto Ballester ${ }^{* * *}$ \\ Jorge Guedes Silveira ${ }^{* * * * *}$ \\ Fladhimyr Câmara Castello ${ }^{* * * * *}$
}

Resumo. Este artigo descreve o projeto de um software educacional que tem como objetivo promover o conhecimento científico através da utilização de uma ferramenta on-line para estudos de casos clínicos, disponibilizando opções de busca, visualização e discussão de informações em saúde. Em sintonia com as tendências atuais da educação médica, o sistema MedCase $e^{1}$ proporciona aos estudantes e professores da Faculdade de Medicina (PUCRS) um ambiente para o conhecimento da realidade, dos problemas de saúde mais prevalentes e prioritários da população brasileira e, através de estudos de casos clínicos, simular a solução destes problemas.

Palavras-Chave. casos clínicos, informática na saúde, informática na educação, aprendizagem baseada em problemas

Abstract. This paper describes an educational software project to promote the scientific knowledge through an on-line tool for clinical cases studies, with search, viewing and discussion options. The present medical education tendencies, the system MedCase provides for students and professors of the Medicine Faculty (PUCRS) an environment for reality knowledge, priority health problems of the Brazilian population and, trough of clinical cases studies, simulate the problems solution.

Keywords. clinical cases, health technology, education technology, problem based learning

\section{Introdução}

Não é possível ensinar, ou aprender medicina, sem prestação de assistência médica. Deste modo, ensino e assistência estão intimamente ligados e se confundem a partir de um determinado nível da graduação. Porém, muitas vezes, alguns princípios éticos, tais como não maleficiência e privacidade, podem dificultar o ensino, na medida em que a assistência é feita por um estudante, mesmo que este esteja sob orientação direta do professor.

\footnotetext{
* Doutoranda PPGC/UFRGS. Pesquisadora do Grupo de Pesquisas Avançadas em Redes de Comunicação (GPARC) da PUCRS. hmelo@gparc.org, www.gparc.org.

** Mestrando PPGEE/PUCRS. Pesquisador do Grupo de Pesquisas Avançadas em Redes de Comunicação (GPARC) da PUCRS. lbasso@gparc.org, www.gparc.org.

*** Professor, Phd, Coordenador do Laboratório de Tecnologias Educacionais em Saúde da PUCRS. dinarte@pucrs.br, http://www.pucrs.br/famed/lates.

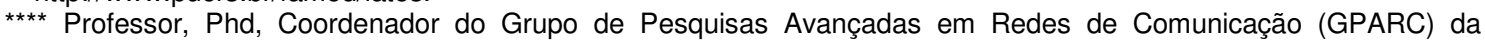
PUCRS.jguedes@ee.pucrs.br, www.gparc.org.

***** Doutorando PPGC/UFRGS. Pesquisador do Grupo de Pesquisas Avançadas em Redes de Comunicação (GPARC) da PUCRS. facastello@gparc.org, www.gparc.org.

1 Informações sobre o projeto disponíveis em http://www.gparc.org/emergencyroom

V.3 $\mathrm{N}^{\circ} 2$, Novembro 2005
} 
Tanto durante o período de iniciação à aprendizagem de clínica médica ou no decorrer de praticamente toda a vida do profissional médico, tomam-se como base principalmente a vivência e discussão de casos. Na Faculdade de Medicina da Pontifícia Universidade Católica do Rio Grande do Sul (FAMED-PUCRS), alunos, internos e médicos do programa de residência médica, são treinados, fundamentalmente, através de reuniões clínicas em pequenos grupos ou em grandes sessões. Nessas reuniões, objetiva-se estimular a prática de exercícios de elaboração de hipóteses. As hipóteses, uma vez elaboradas, podem ser confirmadas, ou afastadas, com a ajuda de exames (imagens, gráficos, ou dados bioquímicos). Com esse "roteiro" chega-se, na maioria das vezes, a um diagnóstico definitivo e, portanto, à possibilidade terapêutica.

Resumidamente, isto é o chamado "raciocínio clínico". Essa situação é exaustivamente treinada na apresentação de casos de alta incidência e prevalência na população, ou também, em casos, onde embora não tão freqüentes, apresentam a possibilidade de uma "solução dramática" (cura ou salvamento), quando diagnosticados.

As sessões de discussão de casos acima citadas costumam apresentar uma falta de flexibilidade referente às datas e locais de suas realizações, uma vez que elas são promovidas em dias e horários específicos, bem como em um lugar predeterminado. Muitas vezes alunos e demais profissionais interessados não dispõem da possibilidade de participar destas sessões dentro destes termos, o que acaba por excluí-los deste processo de enriquecimento do conhecimento.

Outro problema observado se refere à permanência das informações discutidas nas sessões e ao acesso às mesmas no período posterior a sua realização. Muitas discussões ali debatidas acabam por se perder com o passar do tempo, uma vez que ficam armazenadas apenas na memória dos indivíduos participantes da sessão. Não existem meios para retomada dos assuntos abordados e dos diálogos gerados com base neles, tanto para os participantes quanto para aqueles indivíduos que não tiveram a oportunidade de estar presente no momento da sua realização.

Observamos que o desenvolvimento de ferramentas informatizadas para possibilitar o auto-aprendizado através da simulação das reuniões de discussão de casos clínicos realizadas dentro da faculdade, pode vir a ser um instrumento de grande ajuda no aprendizado médico continuado, suprimindo as carências acima citadas.

Tendo como base esta motivação, foi realizada uma análise de diferentes ambientes que oferecem recursos para a análise de casos clínicos. Esta análise teve como objetivo identificar os pontos positivos e as carências, utilizando estas informações como referências na construção de uma nova proposta. Entre os projetos estudados, pode-se destacar: Hospital Virtual Brasileiro (2005), The Interactive Patient (2005), CyberPatient Simulator (2005), Virtual Hospital (2005), Sociedade Brasileira de Nefrologia (2005), SisMe (Gastal, Ballester e Rodrigues, 2001) e CardioCaseDiscussion (Moreira 2002).

Percebe-se, através desta avaliação, a ausência de estruturação das informações na grande maioria dos ambientes, bem como uma carência de ferramentas de cooperação, as quais possam armazenar informações construídas coletivamente pela interação dos usuários.

A investigação sobre as necessidades dos indivíduos envolvidos no processo de discussão dos casos e as carências das propostas estudadas nos levaram ao desenvolvimento de um software educacional, denominado MedCase, o qual tem por objetivo possibilitar aos alunos de medicina o estudo de casos clínicos de alta prevalência na nossa população, complementando o ensino presencial atualmente instituído e rompendo barreiras espaço-temporais que pudessem vir a dificultar o processo de aprendizagem. 
O presente artigo está estruturado em cinco seções. Na segunda seção é apresentado o sistema MedCase de forma detalhada, destacando seus objetivos, funcionalidades, cenários de aplicação e arquitetura. A terceira seção descreve as tecnologias de desenvolvimento utilizadas e na quarta seção são destacados os resultados obtidos na primeira fase do projeto. Finalmente, na seção cinco são abordadas as nossas conclusões, seguindo os agradecimentos e referências bibliográficas.

\section{Projeto do Sistema MedCase}

Andrade (2001) destaca que o desenvolvimento de ambientes virtuais de ensinoaprendizagem necessita previamente de um conjunto de estratégias pedagógicas, as quais devem ser definidas levando em consideração os objetivos e pressupostos pedagógicos do professor ao qual a ferramenta visa atender. Deve-se, portanto, inicialmente definir uma arquitetura pedagógica a ser utilizada para o ambiente, para só então partir para as definições tecnológicas e o desenvolvimento do sistema.

A partir desta necessidade, optamos pela utilização do PBL (Problem Based Learning) como base para o desenvolvimento do MedCase, uma vez que este método "busca a integração das disciplinas clássicas em módulos de ensino, com temática específica, trabalhada a partir da discussão de problemas e busca de informações e subsídios teóricos e técnicos para sua solução" (Kodjaoglanian 2003, p.4).

A ferramenta aqui proposta busca atender a um dos quatro grandes eixos do PBL destacados por Venturelli (2003): o estudo com base em problemas fundamentados na realidade, tendo como conseqüência pesquisa e discussão em grupos para alcançar uma possível solução, bem como compreensão e aquisição de conhecimento.

$\mathrm{O}$ projeto do sistema MedCase tem como objetivo oferecer à comunidade médica e acadêmica uma ferramenta a ser utilizada como fator de qualificação nos estudos de casos clínicos. Através do MedCase, são disponibilizados diferentes casos fictícios, criados a partir de experiências ou contextos relevantes da nossa realidade, possibilitando que os alunos realizem a linha de raciocínio necessária para chegar ao diagnóstico e ao respectivo tratamento e prognóstico para um determinado paciente.

Para contemplar todas as informações necessárias para apresentar uma visualização clara e concisa sobre o caso clínico do paciente a ser analisado, dividiu-se o mesmo em frações menores conforme é apresentado na Figura 1.

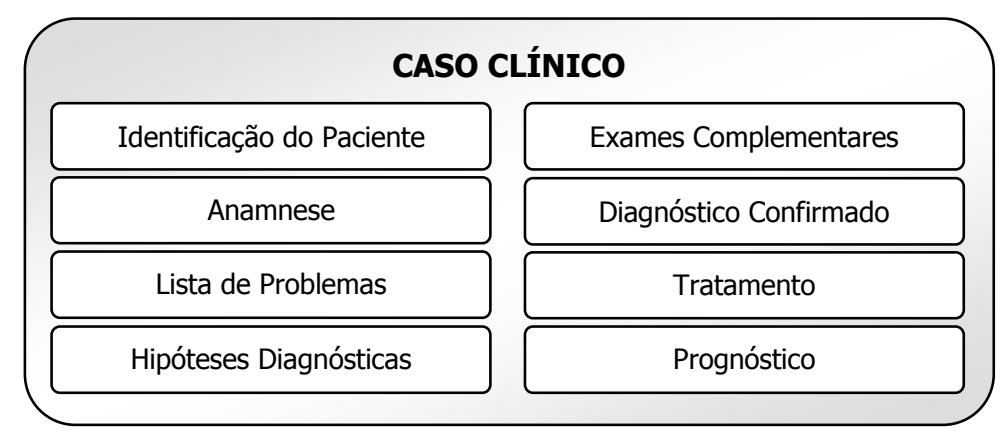

Figura 1. Estrutura do Caso Clínico

A Identificação do Paciente possibilita aos estudantes conhecer o paciente que será estudado. Contempla informações sobre a sua idade, sexo, cor, estado civil, naturalidade e procedência, grau de escolaridade, profissão e ocupação atual, além de outras informações que dizem respeito à pessoa do paciente. As informações clínicas do paciente são apresentadas na Anamnese através dos seguintes itens: Queixa Principal, 
História Médica Atual do Paciente, Revisão de Sistemas, História Médica Pregressa, Hábitos, História Psicossocial, Exame Físico e Impressão sobre o Paciente.

Após a leitura da Identificação e da Anamnese, os estudantes podem levantar os problemas encontrados para o paciente em estudo, sendo essa Lista de Problemas a base para a elaboração dos possíveis diagnósticos. As Hipóteses Diagnósticas são elaboradas primeiro e consideram o conjunto de alterações no organismo que tem relação entre si (Hipóteses Diagnósticas Sindrômicas) e os possíveis agentes causadores da doença (Hipóteses Diagnósticas Etiológicas).

Com o auxílio dos Exames Complementares, os estudantes irão avaliar as suas hipóteses para finalmente chegar ao seu Diagnóstico Confirmado. Os exames disponibilizados no MedCase são compostos pelos resultados de exames laboratoriais e exames de imagens e traçado.

Uma vez identificado e confirmado o diagnóstico do paciente, os próximos itens a serem trabalhados referem-se ao Tratamento Terapêutico e ao Prognóstico. No Tratamento são definidos dieta, cuidados gerais, orientações para o próprio paciente e para os seus familiares, orientações psico-sociais e o tratamento farmacológico. No Prognóstico encerra-se o caso com a previsão da evolução clínica do paciente. Toda a linha de raciocínio realizada pelos autores do caso clínico pode ser consultada pelos estudantes durante o estudo.

O MedCase foi projetado para disponibilizar, além dos próprios casos clínicos, ferramentas complementares com diferentes conteúdos e materiais. Desta forma, médicos especialistas podem inserir opiniões sobre os casos das suas respectivas áreas de atuação, bem como participar dos fóruns de discussão sobre as questões levantadas pelos alunos.

As dúvidas sobre conceitos utilizados para descrever o caso podem ser esclarecidas no Dicionário de Termos, planejado para possibilitar uma rápida pesquisa de termos e significados, bem como para apresentar-se como uma ferramenta de construção colaborativa do conhecimento, na medida em que os usuários podem sugerir novos termos para esta base. Para enriquecer o conteúdo do caso, podem ser consultadas as principais referências bibliográficas dos assuntos abordados, bem como ser realizada a proposta de novas referências para o caso, estimulando assim a interação do usuário.

A Figura 2 apresenta a utilização do sistema MedCase pelos alunos, professores e médicos, destacando as diversas interações possíveis para os diferentes tipos de usuários. 

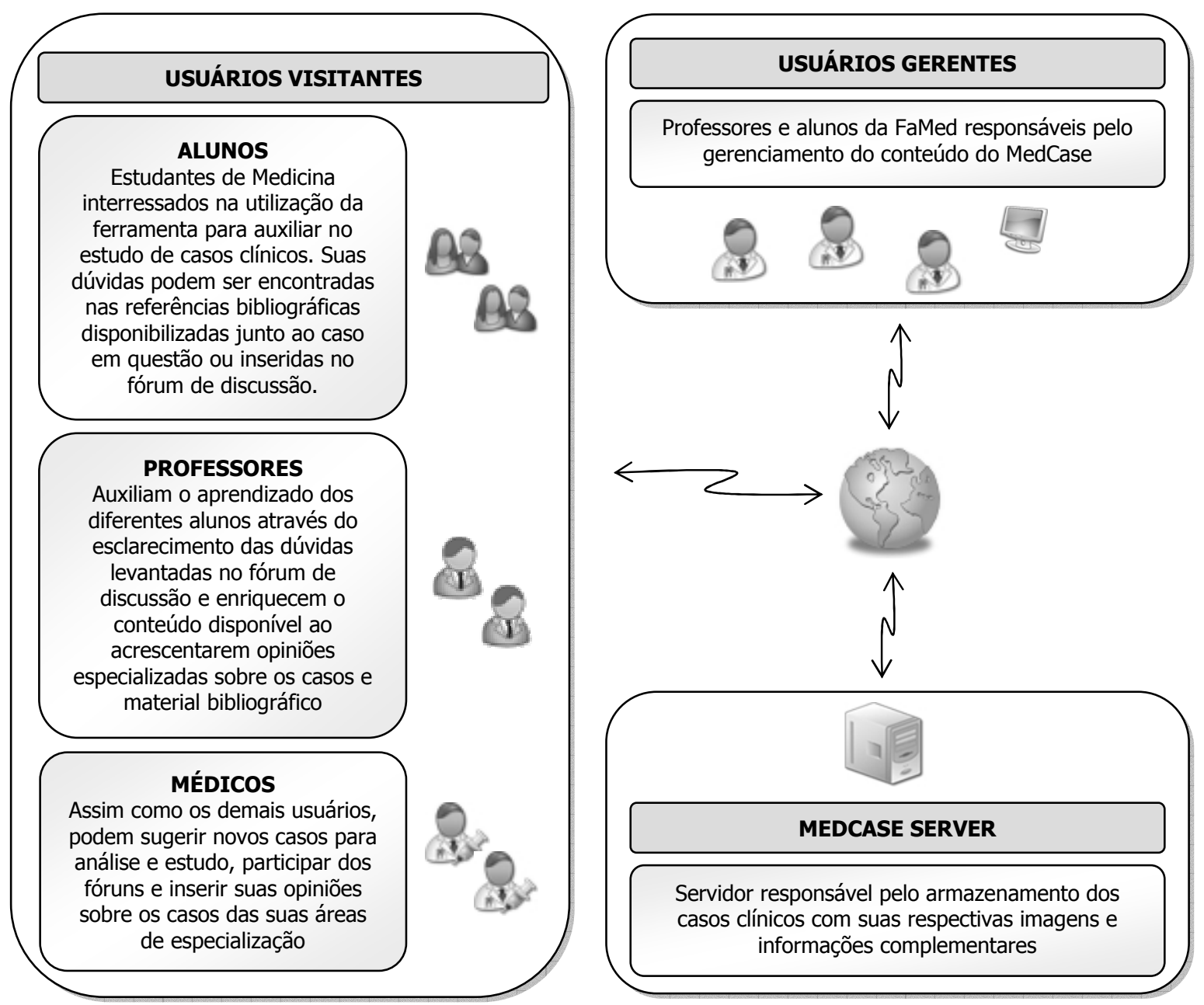

Figura 2. Cenário de Aplicação do MedCase

Conforme apresentado na Figura 2, o sistema considera dois níveis de acesso, com privilégios diferentes, através de dois tipos de usuários: visitantes ou gerentes. Considera-se como usuários Visitantes do sistema MedCase os professores, médicos e estudantes interessados na utilização da ferramenta para auxílio acadêmico. A diferença entre os usuários Visitantes e Gerentes consiste no nível de acesso a determinadas funcionalidades. Ambos podem realizar a atividade fim do MedCase, ou seja, consultar casos clínicos para a realização de estudos. Mesmo a possibilidade de inserção de novos casos no sistema, bem como a alteração desses casos, é disponibilizada a todos os participantes, incentivando a participação de toda a comunidade acadêmica no enriquecimento de novos casos clínicos.

Ao Gerente cabe as atividades de gerenciamento das informações disponibilizadas na ferramenta. Todos os novos casos clínicos sugeridos são anteriormente analisados pelos Gerentes para a verificação da consistência das informações e para autorização de publicação. Após a publicação, os casos são disponibilizados para acesso por todos os interessados. 


\section{Ferramentas de Desenvolvimento}

O MedCase foi projetado sobre um modelo web-based, visando a facilidade de uso, interface amigável, interatividade e independência da localização geográfica. Desta forma, são disponibilizadas interfaces gráficas específicas para acesso, via Internet, aos serviços disponíveis. Para possibilitar o armazenamento das informações (casos clínicos, resultados de exames, opinião de especialistas, entre outras) foi integrado ao sistema a utilização de um banco de dados. Os dados inseridos podem ser alterados, consultados e excluídos, conforme as especificações e níveis de autorização (camada de segurança). Além de possibilitar o armazenamento de dados, é também realizado o armazenamento de imagens que correspondem aos resultados de diferentes exames.

Uma característica importante referente ao trabalho que deve ser destacada é que todo o desenvolvimento do sistema foi baseado na utilização de softwares livres. Foram utilizados o sistema operacional GNU/Linux, o gerenciador de banco de dados MySQL $^{2}$ e o servidor Web Apache ${ }^{3}$ sendo que as diferentes funcionalidades foram implementadas utilizando a linguagem $\mathrm{PHP}^{4}$ como linguagem de programação principal, intercalando com códigos em JavaScript ${ }^{5}$ e HTML ${ }^{6}$.

A metodologia de processo unificado de desenvolvimento de software (RUP - Rational Unified Process ${ }^{7}$ ) foi adotada visando obter um melhor nível de produtividade e qualidade da equipe de desenvolvimento.

\section{Resultados}

Como resultados da primeira fase do desenvolvimento desse projeto, foi obtido um sistema para atender as necessidades básicas para a discussão de casos clínicos. Nesta seção serão apresentadas algumas das inúmeras interfaces desenvolvidas.

$\mathrm{Na}$ interface para a seleção de casos clínicos, conforme mostra a Figura 3, uma lista de casos é disponibilizada com uma pequena identificação do caso. Ao selecionar um determinado caso clínico, na área de apresentação é disponibilizado um pequeno detalhamento do caso para que o estudante conheça brevemente alguns dos problemas a serem estudados.

\footnotetext{
${ }^{2}$ MySQL. Disponível em: <http://www.mysql.org>.

3 Apache Software Foundation. Disponível em: <http://www.apache.org >.

${ }^{4}$ PHP. Disponível em: <http://www.php.net>.

${ }^{5}$ Sun Developer Network. Disponível em: < http://www.mozilla.org/js >.

${ }^{6}$ WC3. Disponível em: <http://www.w3.org/MarkUp>.

${ }^{7}$ Rational Software. Disponível em: <http://www-306.ibm.com/software/rational>.
} 


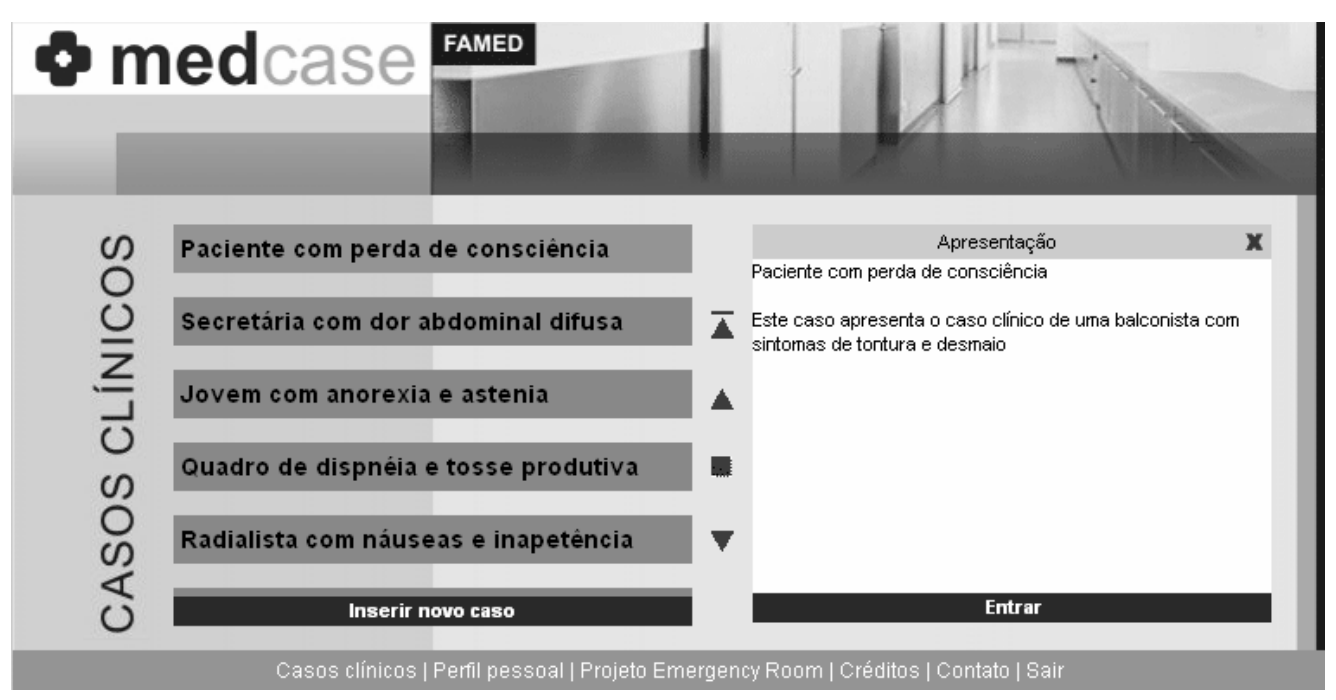

\section{Figura 3. MedCase - Seleção de Casos Clínicos}

Ao selecionar um caso clínico para estudo, pode-se alternar entre os ítens que compõe o caso através do menu esquerdo da interface, conforme mostra a Figura 4 onde são apresentadas as interfaces de Anamense e Lista de Problemas.

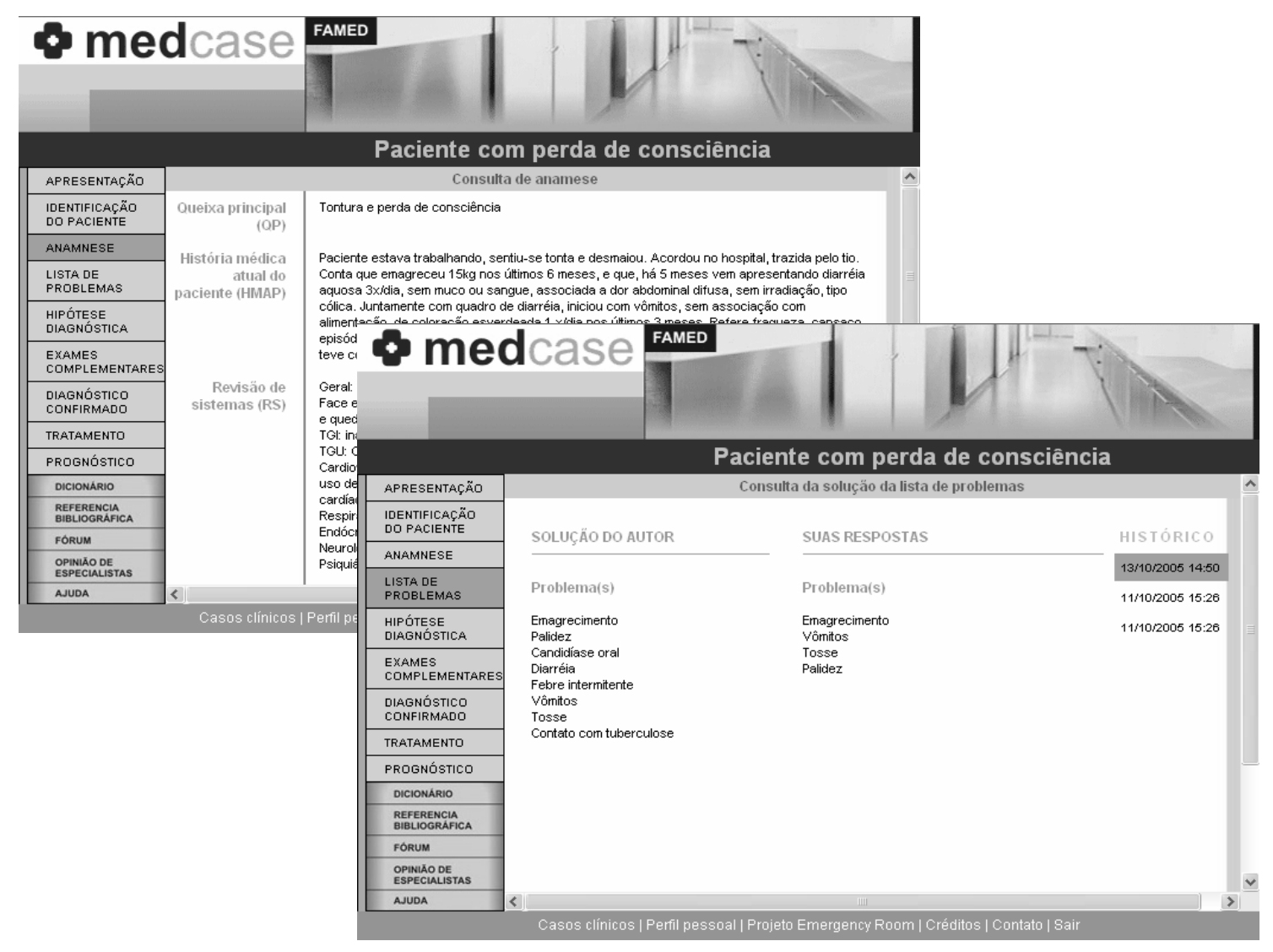

Figura 4. MedCase - Estudo de Casos Clínicos 
$\mathrm{Na}$ Anamnese os estudantes têm acesso à história clínica do paciente e pode ser consultada a qualquer momento durante o estudo do caso. As informações disponibilizadas na Identificação do Paciente e na Anamense são disponíveis apenas para consulta.

No que se refere à Lista de Problemas, tanto nela quanto nos Diagnósticos, Tratamento e Prognóstico, todas as interações dos usuários são armazenadas na forma de históricos (identificados pela data/hora) para possibilitar uma visão da linha de raciocínio realizada para chegar ao resultado final. Ao concluir as suas próprias respostas, o estudante pode compará-las com as soluções propostas pelos autores do caso.

Complementado o caso, são disponibilizados acesso para as ferramentas de Dicionário de Termos, Referências Bibliográficas, Fórum de Discussão e Opinião de Especialistas, sendo o conteúdo direcionado conforme o caso clínico selecionado.

\section{Conclusão}

O desenvolvimento do sistema MedCase foi proporcionado através de uma ação realizada pelo PROMED (Programa de Incentivo às Mudanças Curriculares para as Escolas Médicas). O MedCase é resultado dos esforços conjuntos de uma equipe multidisciplinar composta por alunos e professores tanto da Faculdade de Medicina quanto da Faculdade de Engenharia, ambos da PUCRS.

Durante a fase de modelagem da ferramenta, buscou-se planejá-la de forma que as informações componentes de um caso clínico fossem apresentadas de uma maneira estruturada. Além de focar na estruturação das informações, buscou-se oferecer meios para que os usuários pudessem realizar a interatividade entre si, explicitando seus questionamentos sobre o caso e discutindo os assuntos que julgarem relevantes para que, através da cooperação mútua, consigam chegar a um consenso ou resultado. A utilização de ferramentas cooperativas de armazenamento do conhecimento (dicionários, referências bibliográficas e casos) também tem destaque nesta proposta.

Como trabalhos futuros destaca-se a avaliação da utilização do MedCase como ferramenta educacional na Faculdade de Medicina, comparando os estudos de casos clínicos presenciais com os estudos realizados utilizando esta ferramenta web-based.

Considerando as tendências na área da educação médica, o MedCase representa uma ferramenta aberta para a comunidade médica acadêmica ao viabilizar a união de conhecimento científico com ambientes tecnológicos, permitindo a inserção contínua de novos casos por estudantes e professores. Ao apresentar-se como uma proposta de código livre, permite-se uma constante evolução da ferramenta através de iniciativas da comunidade de software livre, sendo possível o desenvolvimento de núcleos específicos para sua aplicação a outras áreas do conhecimento, uma vez que o método PBL é perfeitamente aplicável ao ensino em qualquer outro ramo do saber (Kodjaoglanian, 2003).

\section{Agradecimentos}

Agradecemos ao Ministério da Saúde e ao Ministério da Educação pela disponibilização de verbas ao Programa de Incentivo às Mudanças Curriculares para as Escolas Médicas (PROMED) da Faculdade de Medicina (PUCRS).

\section{Referências Bibliográficas}

ANDRADE, Adja Ferreira de et al. Requisitos para a Modelagem de Ambientes de Aprendizagem a Distância: uma Proposta da PUCRS VIRTUAL. In: International Conference On New Technologies In Science Education, 2001, Aveiro, Anais... Aveiro, Portugal. 2001. 
CYBERPATIENT Simulator. Desenvolvido pela MDChoice.com. Disponível em: $<$ http://www.mdchoice.com/cyberpt>. Acesso em: 10 set. 2005.

GASTAL, F. L.; BALLESTER D.A.; RODRIGUES D. S. Sistema de Informações em Saúde Mental (SISMe): uma ferramenta de educação à distância para os serviços básicos de saúde. In: Congresso da Rede UNIDA, 4., 2001, Londrina. Anais... Londrina: Rede Unida, 2001.

HOSPITAL Virtual Brasileiro. Desenvolvido pelo Núcleo de Informática Biomédica Unicamp. Disponível em: <http://www.hospvirt.org.br>. Acesso em: 15 set. 2005.

INTERACTIVE Patient. Desenvolvido pela Marshall University - Division Information Technology and Medical Informatics. Disponível em: <http://medicus.marshall.edu>. Acesso em: 10 set. 2005.

KODJAOGLANIAN, Vera Lucia; et al. Inovando Métodos de Ensino-Aprendizagem na Formação do Psicólogo. Psicologia Ciência e Profissão, v. 23, n. 1, p. 2-11, 2003.

MOREIRA, Gláucia de Sá Fortes; et al. CardioCaseDiscussion: um ambiente virtual de aprendizagem cooperativa. In: Simpósio Brasileiro de Informática na Educação, 13., 2002, São Leopoldo. Anais... São Leopoldo: UNISINOS, 2002.

SOCIEDADE Brasileira de Nefrologia. Disponível em: <http://www.sbn.org.br/index1.html>. Acesso em: 15 set. 2005.

VENTURELLI, José. Educacion médica: Nuevos Enfoques, Metas y Métodos. Organización Panamericana de la Salud. Salud y Sociedad, n. 8, p. 20-91, Washington DC: Edit Paltex. 2003.

VIRTUAL Hospital. Desenvolvido pela University of Iowa. Disponível em: $<$ http://www.vh.org>. Acesso em: 15 set. 2005. 